\section{Glaucoma surgery in the United Kingdom: why, who and when}

The group of diseases known as glaucoma will be the major cause of irreversible blindness in the world by the year $2000 .{ }^{1}$ This group of patients also account for up to one-third of all patients who are seen in ophthalmic clinics in the United Kingdom. There are very few ophthalmologists who will be able to ignore the burdens glaucoma will impose upon their ophthalmology service.

We should therefore all turn to the national survey of trabeculectomy in the United Kingdom published by Edmunds, Thompson, Salmon and Wormald in this issue of Eye. ${ }^{2}$ This is one of a series of important studies sponsored by the Department of Health and implemented through the Royal College of Ophthalmologists. This study is unique in that there are, as far as we know, no other prospective national surveys of trabeculectomy anywhere else in the world. Therefore, it helps us all to know what is happening in the 'real world' in the United Kingdom, and will stand as a landmark study for that reason. Although the UK National Health system is sometimes criticised, this unified system does facilitate this type of important study.

Most ophthalmologists in the United Kingdom carry out trabeculectomy. Although much less commonly carried out than cataract surgery, trabeculectomy is still one of the more commonly performed intraocular operations in the United Kingdom, with approximately 20000 operations being carried out each year. ${ }^{3}$ Therefore, if the data in this survey can help identify areas where improvements can be made nationwide, then this could potentially have a very significant impact on many thousands of our patients. So what can we learn from the survey?

Most of the patients in the survey had primary open angle glaucoma $(89.2 \%)$, with the rest having pseudoexfoliation $(5.4 \%)$, normal tension glaucoma $(3.8 \%)$ and pigmentary dispersion syndrome $(1.6 \%) .^{2}$ Other forms of glaucoma were excluded. In other parts of the world the relative importance of different diagnoses such as pseudoexfoliation and angle closure glaucoma would be expected to be very different. This emphasises the importance of having specific studies for different parts of the world.

The patient demographics are also important. The mean age of the patients was 69.2 years and $2.1 \%$ died after 1 year of followup. Although this rate would be expected to increase based on actuarial tables, if this trend continued a large number of patients would survive over 10 years. This reiterates the importance of achieving continued success of the trabeculectomy surgery in the long term to preserve vision and quality of life for these patients. This is particularly the case as $50.5 \%$ of the patients had advanced field loss at the time of listing for surgery, and failure of medical treatment was cited as the reason for surgery in $87 \%$ of the patients. Failure of surgery and poor intraocular pressure control would be expected to lead to visual loss in a relatively short time in these eyes with advanced disease. ${ }^{4}$

The reasons for surgery (more than one response allowed) included inadequate intraocular pressure control $(87 \%)$, deteriorating visual fields (48.8\%) and progressive optic nerve damage $(26.4 \%)$. Although visual field and optic nerve assessment occupy significant health care resources this highlights the importance of having adequate and accurate systems of recording and measuring change in these parameters to ensure appropriate surgical decisions are made. The need for accurate field and disc assessment is further supported by the fact that only $35.3 \%$ of the patients had advanced visual field damage at the time of diagnosis. After a median time of 2.7 years follow-up, 50.5\% had advanced visual field damage. Therefore we need to be able to detect or even predict significant deterioration more rapidly to avoid this situation. ${ }^{5}$

There is a perception abroad that primary surgery (surgery at the time of diagnosis) is carried out in the United Kingdom. This is mainly due to the studies in Cambridge, ${ }^{6}$ Glasgow $^{7}$ and Moorfields. ${ }^{8}$ However, only $4.8 \%$ of surgeons reported that they operated because they carried out primary surgery. This low incidence of primary surgery is supported by the fact that the authors of the survey estimate
P.T. Khaw

D. Siriwardena

Institute of Ophthalmology and Moorfields Eye Hospital London, UK

Professor P.T. Khaw, PhD, FRCP, FRCS, FRCOphth Glaucoma and Wound Healing Research Units Institute of Ophthalmology and Moorfields Eye Hospital 11-43 Bath Street London EC1V 9EL, UK

P.T.K. and D.S. are supported by the Medical Research Council (UK) G9330070 
that only $4 \%$ of glaucoma patients in the United Kingdom undergo trabeculectomy. This means that the majority of patients received medical treatment. Fifty per cent of the patients were on two medications, with $48.5 \%$ having been on pilocarpine and/or adrenaline at some stage prior to surgery.

This has potentially large implications for the success of trabeculectomy in the United Kingdom. Primary surgery studies in the United Kingdom have shown success rates of approximately $90 \%$, but this has not been the experience of many other centres where patients have received prior medical treatment. Broadway et al. ${ }^{9}$ showed a 'success rate' of $93 \%$ with beta-blockers alone, falling to $72 \%$ with beta-blockers and miotics, and $45 \%$ with beta-blockers, miotics and adrenaline. If the findings of Broadway's study apply to the UK patients, a difference in success between $93 \%$ and $72 \%$ would mean an extra 4200 patients 'failing' filtration surgery in the United Kingdom. If the success rate which will be reported in subsequent reports from this survey is closer to the lower figure, this makes the question as to whether we should use an antimetabolite with all trabeculectomies in the United Kingdom even more pertinent. ${ }^{10}$ Pilocarpine and adrenaline have been largely superseded by a new generation of topical medications, but further studies are needed to determine whether these new medications have any effect on the conjunctival healing response.

Finally, given the long-term nature and the importance of glaucoma, it is to be hoped that this survey will continue to be funded, and the patients followed in a further longer-term study in the future. In the meantime we all await the next paper with the results of trabeculectomy surgery in the United Kingdom with great interest.

\section{References}

1. Thylefors B, Negrel A-D, Parajasegaram R, Dadzie D-Y. Global data on blindness. Bull WHO 1995;1:115-21.

2. Edmunds B, Thompson JR, Salmon JF, Wormald RP. The national survey of trabeculectomy. I. Sample and methods. Eye 1999;13:524-30.

3. Department of Health Hospital Episodes Statistics Database. London: Department of Health, 1994/5.

4. Grant WM, Burke JF. Why do some people go blind from glaucoma? Ophthalmology 1982;89:991-8.

5. Crabb DP, Fitzke FW, McNaught AI, et al. Improving the prediction of visual field progression in glaucoma using spatial processing. Ophthalmology 1997;104:517-24.

6. Watson PG, Grierson I. The place of trabeculectomy in the treatment of glaucoma. Ophthalmology 1981;88:175-96.

7. Jay JL, Murray SB. Early trabeculectomy versus conventional management in primary open angle glaucoma. $\mathrm{Br} \mathrm{J}$ Ophthalmol 1988;72:881-9.

8. Migdal C, Hitchings RA, Gregory W. Primary treatment trial analysis of visual fields. Eye 1993;114:737-41.

9. Broadway DC, Grierson I, O’Brien C, Hitchings RA. Adverse effects of topical anti-glaucomatous medication regimens. II. Effects on the outcome of filtration surgery. Arch Ophthalmol 1994;112:1446-54.

10. Khaw PT. Antimetabolites for all? Eye 1997;11:764-6. 